\title{
The Association of Cardiovascular Diseases and Diabetes Mellitus with COVID-19 (SARS-CoV-2) and Their Possible Mechanisms
}

\author{
Sourav Roy ${ }^{1} \cdot \operatorname{Tanoy}_{\text {Mazumder }}{ }^{1} \cdot$ Sujan Banik ${ }^{1}$ (i)
}

Accepted: 20 June 2020 / Published online: 25 June 2020

(C) Springer Nature Switzerland AG 2020

\begin{abstract}
Coronavirus disease 2019 (COVID-19) has become a global concern and public health issue due to its higher infection and mortality rate; particularly, the risk is very higher among the patients who have cardiovascular diseases (CVD) and/or diabetes mellitus (DM). In this review, we analyzed the recently published literature on CVD and DM associated with COVD-19 infections and highlight their association with potential mechanisms. The findings revealed that without any previous history of CVD, the COVID-19 patients have developed some CVD complications like myocardial injury, cardiomyopathy, and venous thromboembolism after being infected with the severe acute respiratory syndrome coronavirus 2 (SARS-CoV-2) and required for those patients an emergency clinical support to be aware to manage those complications. Though the association between DM and COVID-19-induced severe complications is still unclear, the limited data predict that different markers like interleukin (IL)1, IL-6, C-reactive protein, and D-dimer linked with the severity of COVID-19 infection in diabetic individuals. Further studies on a large scale are urgently needed to explore the underlying mechanisms between CVD, DM, and COVID-19 for better treatment.
\end{abstract}

Keywords Cardiovascular diseases · Coronavirus · COVID-19 · Diabetes mellitus: SARS-CoV-2

\section{Introduction}

Coronaviruses are a single-stranded enveloped RNA virus belonging to the Coronaviridae family (beta genus type), have a diameter of 60-140 nm, known to cause respiratory infections in human [1,2]. At the end of 2019, China has experienced an outbreak of a novel coronavirus that killed more than a thousand and infected over 7000 individuals within the first 50 days and later spread rapidly all over the world [3]. At that time initially, it was named novel coronavirus 2019 (2019$\mathrm{nCoV}$ ) by the Chinese Center for Disease Control and Prevention, which was further renamed to severe acute respiratory syndrome coronavirus-2 (SARS-CoV-2) by the World Health Organization (WHO) and the disease caused by this SARS-CoV-2 named as coronavirus disease 2019 (COVID19) by WHO [4]. It has around $70 \%$ similar genetic sequence

This article is part of the Topical Collection on Covid-19

Sujan Banik

pharmasujan@yahoo.com

1 Department of Pharmacy, Noakhali Science and Technology University, Noakhali 3814, Bangladesh with 2002-2003 pandemic severe acute respiratory syndrome coronavirus (SARS-CoV) [5]. Fever, dry cough, and fatigue are identified as the common symptoms of the infections as well as prolonged prothrombin time and lymphopenia are identified as common characteristics of the infections [6]. The transmission route for COVID-19 to humans is through bat and the intermediate host for transmission is still under research [6], as the virus used an intermediate host to acquire a series of genetic mutations to infect humans effectively [4]. The local transmission of COVID-19 occurred through respiratory droplets, saliva, feces, and urine from infected individuals [4]. In March 2020, the WHO officially declared it as a global pandemic [7]. As of 21st April 2020, 0.16 million deaths with COVID-19 have been reported among 2.4 million confirmed cases globally [2].

Several clinical studies have reported cardiovascular diseases (CVD) as the most common cause of comorbidity in patients with COVID-19; also, a proportion of those patients became critically ill and dead [8], besides, the disease itself is associated with CVD complications [9]. As CVD complications can be a significant contributor to mortality among the patient with the disease, awareness of emergency clinicians becoming very vital [7]. Studies also reported higher risk of a diabetic individual to get infected with the SARS-CoV-2 
virus and having other complications or may die from the disease [2].

In this study, we tried to cover the association of COVID19 infection in individuals with CVD, DM, and their related complications associated with COVID-19 infection.

\section{CVD and COVID-19}

\section{COVID-19 with Pre-existing CVD}

Severe symptoms of COVID-19 are more common in patients with pre-existing CVD when became infected; therefore, a large proportion of deaths from COVID-19 have resulted among those patients [10]. A meta-analysis of 1527 patients with COVID-19 showed that patients with cardiac disease (16.4\%) and hypertension (17.1\%) were more likely to require intensive care [7]. Another study conducted on 44,672 patients with COVID-19 showed that fatality rate is nearly five-fold higher in patients with pre-existing CVD compared to patients without CVD (10.5\% vs. 2.3\%) [7]. In a study on 191 patients from Wuhan, China, $48 \%$ of patients had comorbidity (67\% died), $30 \%$ of which had hypertension ( $48 \%$ died), and $8 \%$ of them had CVD (13\% died) [11]. Similar findings had been suggested by other studies that high risk of mortality in patients with pre-existing CVD [6, 8-10]. Studies reported that patients with hypertension and CVD have higher expression of angiotensin-converting enzyme-2 (ACE2), which could be a potential reason for enhancing susceptibility of those patients to SARS-CoV2 , a possible mechanistic pathway of severity between COVID-19 and CVD (Fig. 1) [9]. Alongside, patients with CVD are more likely to have hypercoagulability due to increase level of D-dimer, which increases the risk of pulmonary embolism, therefore, hypoxia and heart failure [8]. Also, in patients with the acute coronary syndrome (ACS), reduced cardiac function has been observed leading to myocardial ischemia, which might act as a factor to worsen the condition of patients with COVID-19 and may lead to death [10].

\section{CVD Induced by COVID-19}

Angiotensin-converting enzyme 2 (ACE2), a membranebound aminopeptidase, identified as a functional receptor for SARS-CoV-2 [10]. The spike protein of SARS-CoV-2 binds to ACE2 and those trigger the infection which is highly expressed in lungs and heart [10]. The angiotensin II converted from angiotensin I by ACE, responsible for vasoconstriction, increased blood pressure and vascular remodeling. Clinical observation revealed significant elevation of plasma angiotensin II in a patient with COVID-19, which suggests that binding of COVID-19 to ACE2 causes a release of excessive angiotensin II through the renin-angiotensin system (RAS), increasing heart loading, cardio-myocyte hypertrophy, and high blood pressure [1]. Invasion of the virus to the lung surface cells and cardio-myocytes causes inflammation and release of pro-inflammatory cytokines such as interleukin (IL) 1-6, tumor necrosis factor, interferon, endothelial adhesive factor, granulocytes colony-stimulating factor, chemoattractant protein 1 , by the over-activating immune response of the body and increased apoptosis of myocardial cells which enhance the risk of cardiovascular events [1]. Besides the activity of pro-inflammatory cytokines, the myocardial dysfunction could be caused by severe lung failure because of the infection and reduced the amount of oxygen supply or hypoxemia [12].

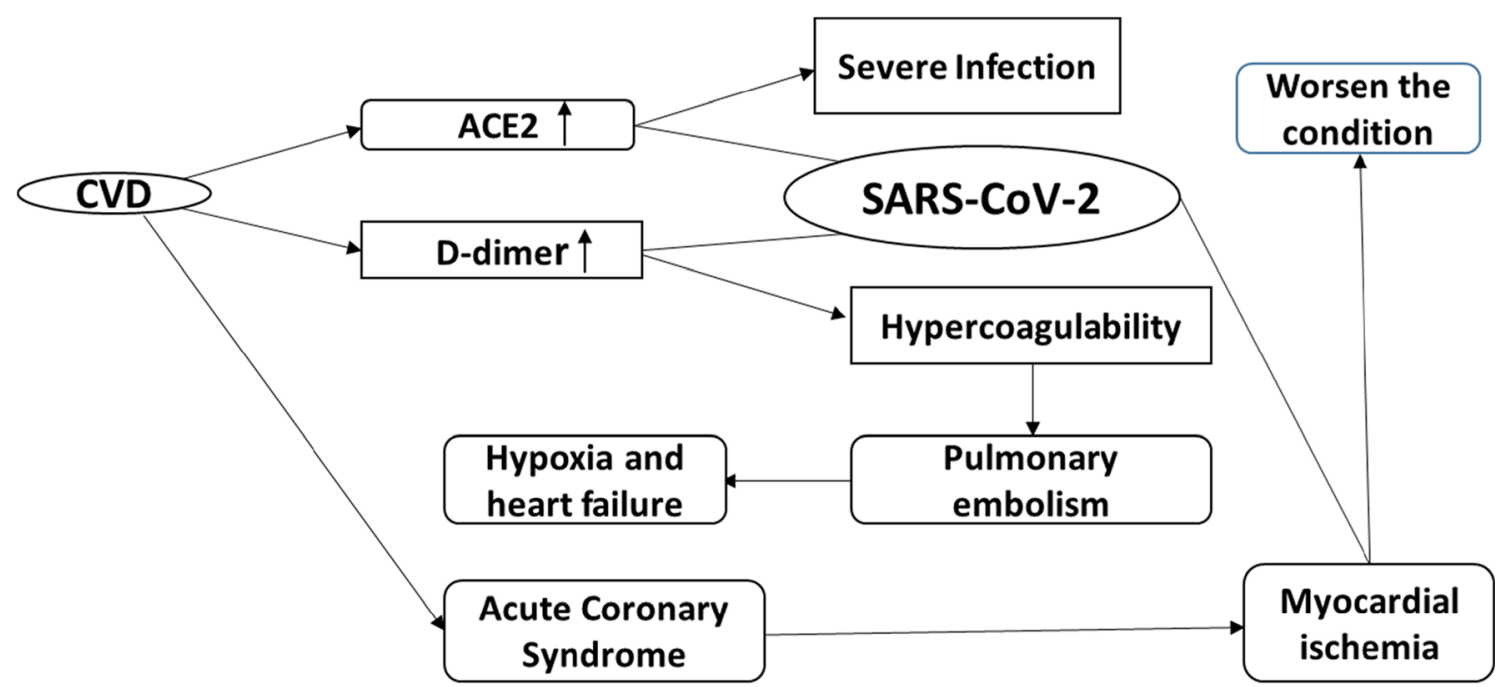

Fig. 1 A possible mechanistic pathway of severity between CVD and COVID-19 and role of ACE2 in COVID-19 
CVD Complications Associated with COVID-19

\section{Myocardial Injury and Myocarditis}

A study performed on 53-year-old white women without any previous history of CVD, admitted to hospital with COVID19 infection and found to have severe left ventricular dysfunction [3]. Increased levels of cardiac troponin were observed in that patient which is identified as a sensitive marker for myocardial injury and the cardiac magnetic resonance imaging (MRI) analysis showed to have diffuse edema and acute myocarditis. Moreover, the study suggests that the onset of symptoms a couple of days after influenza-like syndrome indicates potential myocyte dissemination of the virus, therefore, the onset of heart failure [3]. Association between COVID-19 infection and the myocardial injury was first reported in Wuhan, China, in a study of 41 patients with the disease, among which $12 \%$ of patients had a high sensitivity troponin I above the threshold of $28 \mathrm{pg} / \mathrm{ml}$ [7]. According to the report of the National Health Commission of China, in patients who died from COVID-19, 11.8\% of them were without prior CVD; furthermore, those patients had substantial heart damage and elevated level of troponin. Virus infections such as influenza and parvovirus B-19 are described as one of the most common causes of myocarditis [3]. Though the exact mechanism behind myocardial injury induced by SARSCoV-2 is not fully understood, a previous report revealed the presence of the SARS-CoV genome in the heart of $35 \%$ of patients with the infection, which raises the possibility of direct damage of cardiomyocytes by the virus [12]. As both SARS-CoV and SARS-CoV-2 are highly homologous in the genome, they may have the same pathophysiology. Some other mechanism suggests direct myocardial involvement via ACE2 [11]. Studies also report that cytokine storm in patients with COVID-19, due to the imbalance between subtypes of T helper cells, may contribute to myocardial injury [12].

\section{Cardiomyopathy and Acute Heart Failure}

A study reported that heart failure is an initial presentation of COVID-19 in $23 \%$ of patients, with cardiomyopathy in $33 \%$ of patients [7]. According to another study, 24\% of patients had heart failure, and nearly half of those patients did not have any known history of CVD or hypertension. As heart failure could occur due to exacerbation of prior left ventricular dysfunction, the occurrence of new cardiomyopathy in those patients remains unclear [9].

\section{Venous Thromboembolism}

Due to excessive inflammation, immobilization, and diffuse intravascular coagulation (DIC), COVID-19 may lead to venous thromboembolism [13]. Studies revealed significant abnormalities in the coagulation pathway in patients with COVID-19, along with elevated D-dimer [7]. A study performed on 184 intensive care unit (ICU) patients with COVID-19 pneumonia, where those patients received a standard dose of thromboprophylaxis, despite regular systemic thrombosis prophylaxis, $31 \%$ of those patients have thrombotic complications which are remarkably high and $27 \%$ of them have confirmed venous thromboembolic event (VTE) [13]. A study suggests that anticoagulation therapy with low molecular weight heparin may reduce mortality in patients with severe COVID-19 infection [7].

\section{Cardiac Arrhythmias}

Cardiac arrhythmia is another cardiovascular complication in patients with COVID-19 infection [9]. A study revealed the presence of cardiac arrhythmias in 17\% of hospitalized, and $44 \%$ of ICU patients infected with COVID-19 [7]. However, a high prevalence of cardiac arrhythmias may arise from hypoxia, abnormal metabolism, neuro-hormonal, or inflammatory stress induced by COVID-19 infection.

\section{COVID-19 and DM}

Studies suggest that COVID-19-infected individuals with DM are at higher risk for developing complications including death [14], though the association between diabetes and COVID-19-induced severe disease is not clear yet [15]. However, COVID-19-infected individuals with diabetes have shown three times more fatality rate (7.3\%) compared to those without diabetes (2.3\%), reported by the Chinese Centre for Disease after studying 72,314 COVID-19 cases [14].

\section{Mechanism for the Severity of COVID-19 Infection in DM}

Individuals having DM are more susceptible to infections, especially influenza, and pneumonia; therefore pneumococcal and annual influenza vaccination has been recommended for individuals with diabetes (above 2 years of age) [16]. This is because of their compromised immune system, particularly the innate immunity [17]. Also, innate immunity may be affected temporarily by transient hyperglycemia in response to infection. Numerous factors such as hyperglycemia, altered production of cytokine, impaired $\mathrm{T}$ cell-mediated immune response, inhibition of neutrophil chemotaxis, ineffective microbial clearance, and phagocytic cell dysfunction may contribute to immune dysfunction in diabetic individuals, a possible mechanistic pathway of severity between COVID-19 and DM (Fig. 2) [18]. The hypothesis suggests that ACE2 may play a vital role in the severity of COVID-19 infection in diabetic individuals, as the virus utilize ACE2 for invading 


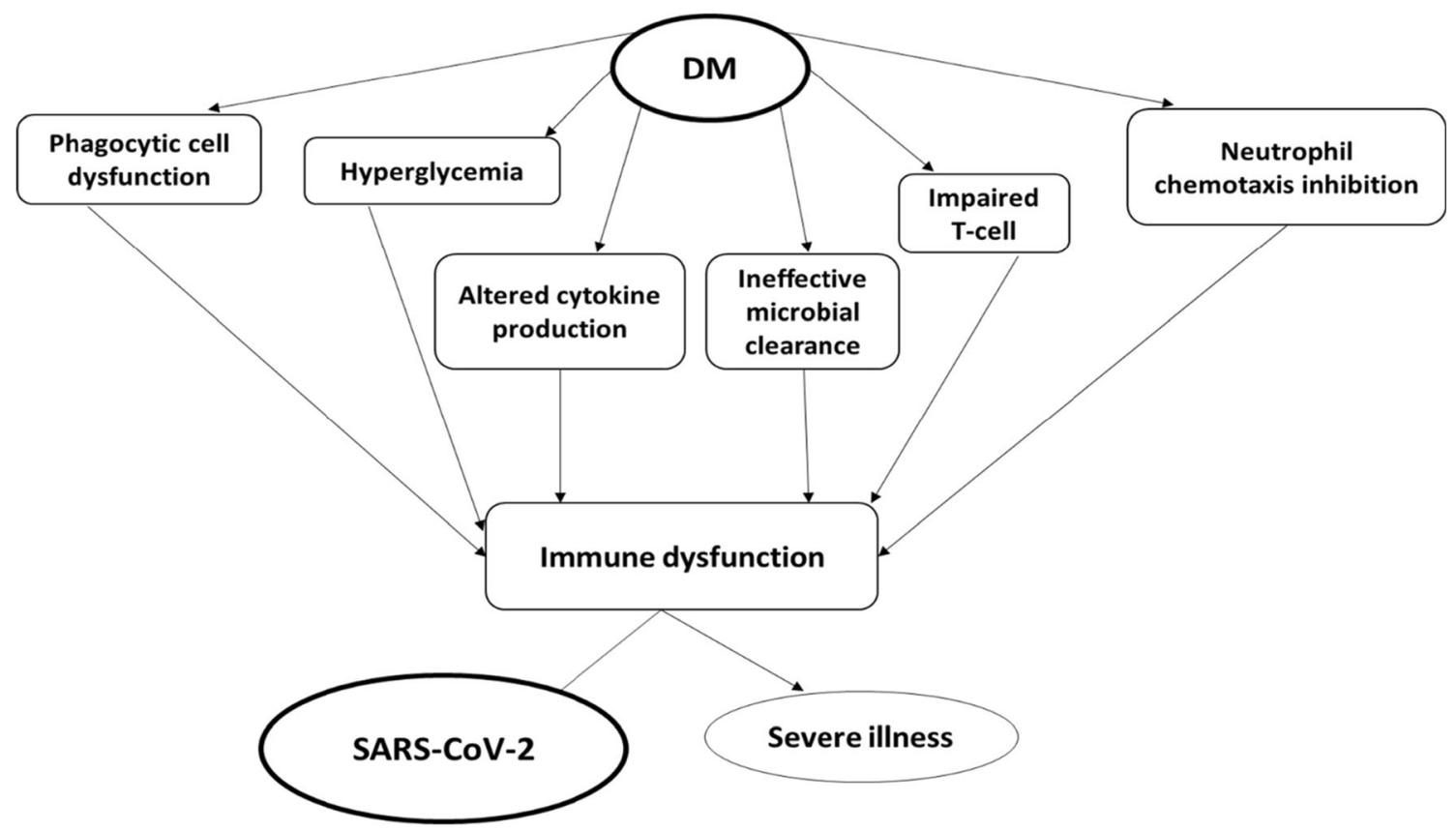

Fig. 2 A possible mechanistic pathway of association between DM and COVID-19

into host pneumocytes [17] and ACE2 is also expressed in pancreatic tissue [18]. Alongside, individuals with diabetes have been shown to have elevated levels of the proinflammatory cytokine, especially IL-1, IL-6, and tumor necrosis factor- $\alpha$ (TNF- $\alpha$ ), and different markers such as Creactive protein, D-dimer, and fibrinogen, those may further prolong the cytokine storms and lead to severe illness in diabetic individuals with COVID-19 infection; the role of the markers in COVID-19 is to induce DM (Fig. 3) [17].
Significant increase in infection and replication of influenza virus after pulmonary endothelial cells being exposed to elevated concentration of glucose in vitro, indicating increased viral replication in hyperglycemic condition. Therefore, hyperglycemia may also play a role in the pathogenesis and prognosis of respiratory viral diseases, though further study needed to conclude this matter [14]. Elevated levels of glucose may also suppress the immune response against the virus. According to experimental data, glycemic

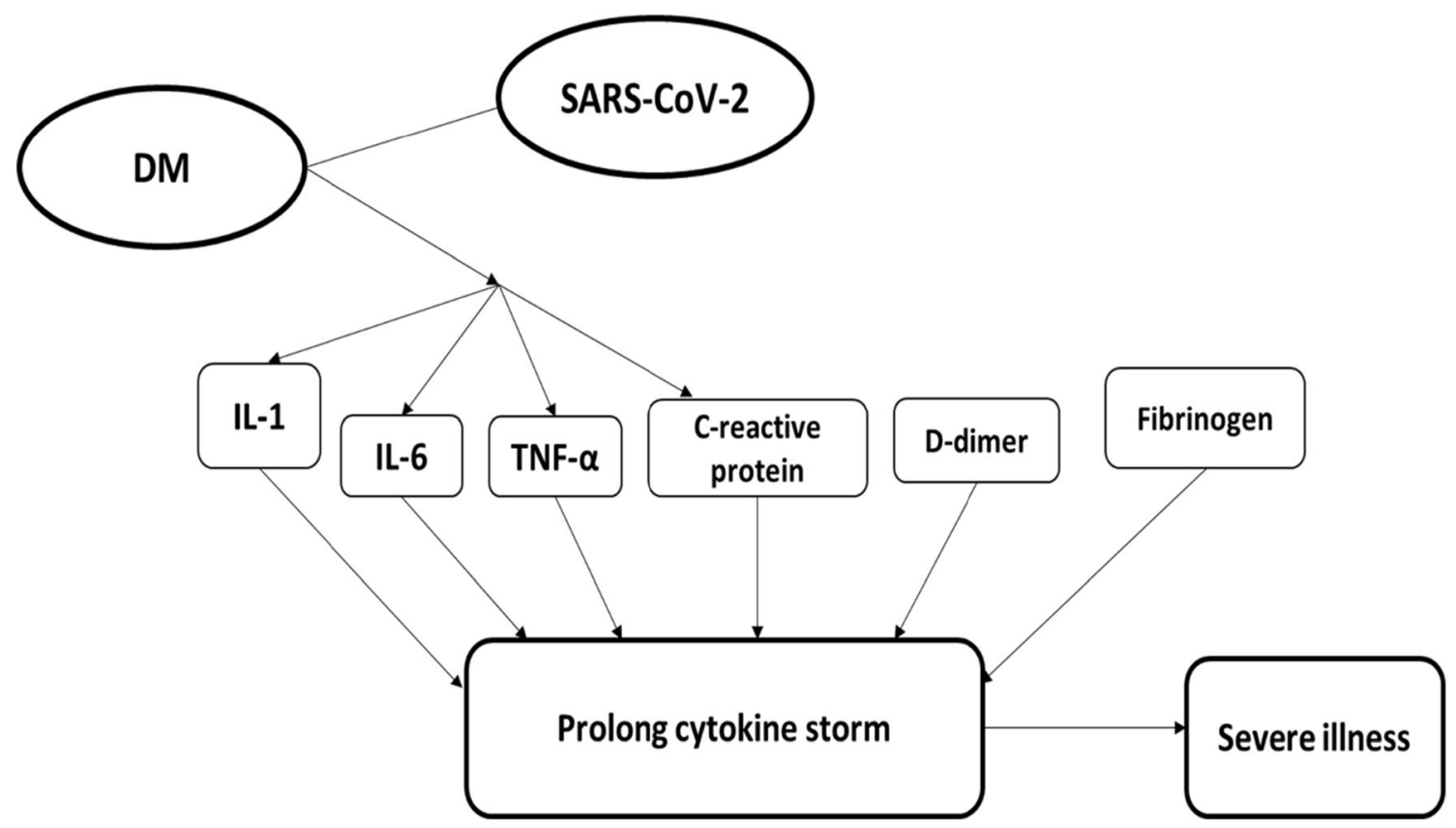

Fig. 3 Role of different marker in COVID-19 to induce DM and their severity 
control can be beneficial clinically in patients who are having both diabetes and respiratory viral infection, like COVID-19 [14].

\section{Medication-Related Risk Factors in Diabetic Individuals with COVID-19}

The SARS-CoV-2 virus attaches to the ACE2 enzyme at low cytosolic $\mathrm{pH}$ and invades into the cell, leading infection [19]. Angiotensin II has a strong role in $\mathrm{pH}$ alkalizing. Angiotensinconverting enzyme inhibitor (ACEI) and angiotensin receptor blockers (ARBs) reduce the levels of angiotensin II and cause low cytosolic $\mathrm{pH}$ [19]. Therefore, diabetes patients who are treated with ACEI and ARBs have shown to have elevated expression of ACE2 and those people have a higher chance to develop severe and fetal COVID-19 infection [20]. A study reported hypoglycemia in a 26-year-old patient with type 1 diabetes after being treated with chloroquine phosphate (500 mg) for 4 consecutive weeks [21]. The use of chloroquine is very frequent in COVID-19-infected patients; therefore, the infected patients with type 1 diabetes should be monitored closely as hypoglycemia could be associated with the increased risk of mortality in those patients. A previous study on SARS-CoV patients without previous diabetes demonstrated pancreatic damage in $51.28 \%$ of patients, leading to acute type 1 diabetes mellitus during hospitalization [14]. This may also be possible in patients with COVID-19 infection, though further evidence is required to conclude this. Alongside, dipeptidyl peptidase-4 (DPP-4) is shown to be the primary receptor of MERS-CoV and there is a chance that the DPP-4 receptor may act as a receptor for COVID-19 infection which is important to explore as DPP-4 inhibitors are widely used in the treatment of diabetes mellitus [14].

\section{Conclusion}

Both the individuals with prior CVD and diabetes are at higher risk to develop severe COVID-19 infection. Alongside, development of CVD complications mostly myocardial injury, cardiomyopathy, venous thromboembolism, and cardiac arrhythmias among the individuals without prior CVD, is also an emerging concern. In diabetic individuals, there are some medications such as ACEI, ARBs, chloroquine, and DPP-4 inhibitors using issues that also arise as some study predicts; their use could worsen the conditions of the patients with COVID-19 which call for the careful use of those medications in diabetic individuals with the infection. Lastly, as the number of infections increasing day by day, it is quite predictable to increase the number of those complicated cases as well. Therefore, medical professional needs to be aware and prepare for managing those emergencies effectively.
Funding Information We did not receive any financial support from any organizations.

\section{Compliance with Ethical Standards}

Conflict of Interest The authors declare that they have no conflict of interest.

Ethical Approval Not required.

Informed Consent No informed consent was necessary.

\section{References}

1. Li G, Hu R, Gu X. A close-up on COVID-19 and cardiovascular diseases. Nutr Metab Cardiovasc Dis. 2020;30:1057-60. https:// doi.org/10.1016/j.numecd.2020.04.001.

2. Muniyappa R, Gubbi S. COVID-19 pandemic, coronaviruses, and diabetes mellitus. Am J Physiol-Endocrinol Metab. 2020;318(5): E736-41.

3. Inciardi RM, Lupi L, Zaccone G, Italia L, Raffo M, Tomasoni D, et al. Cardiac involvement in a patient with coronavirus disease 2019 (COVID-19). JAMA Cardiol. 2020. https://doi.org/10.1001/ jamacardio.2020.1096.

4. Kakodkar P, Kaka N, Baig MN. A comprehensive literature review on the clinical presentation, and management of the pandemic coronavirus disease 2019 (COVID-19). Cureus. 2020;12(4).

5. Song F, Shi N, Shan F, Zhang Z, Shen J, Lu H, et al. Emerging 2019 novel coronavirus (2019-nCoV) pneumonia. Radiology. 2020;295(1):210-7.

6. Li B, Yang J, Zhao F, Zhi L, Wang X, Liu L, et al. Prevalence and impact of cardiovascular metabolic diseases on COVID-19 in China. Clin Res Cardiol. 2020:1-8.

7. Long B, Brady WJ, Koyfman A, Gottlieb M. Cardiovascular complications in COVID-19. Am J Emerg Med. 2020. https://doi.org/ 10.1016/j.ajem.2020.04.048.

8. Li M, Dong Y, Wang H, Guo W, Zhou H, Zhang Z, et al. Cardiovascular disease potentially contributes to the progression and poor prognosis of COVID-19. Nutr Metab Cardiovase Dis. 2020;30:1061-7. https://doi.org/10.1016/j.numecd.2020.04.013.

9. Driggin E, Madhavan MV, Bikdeli B, Chuich T, Laracy J, BiondiZoccai G, et al. Cardiovascular considerations for patients, health care workers, and health systems during the COVID-19 pandemic. J Am Coll Cardiol. 2020;75(18):2352-71.

10. Zheng Y-Y, Ma Y-T, Zhang J-Y, Xie X. COVID-19 and the cardiovascular system. Nat Rev Cardiol. 2020;17(5):259-60.

11. Clerkin KJ, Fried JA, Raikhelkar J, Sayer G, Griffin JM, Masoumi A, et al. Coronavirus disease 2019 (COVID-19) and cardiovascular disease. Circulation. 2020.

12. Guo T, Fan Y, Chen M, Wu X, Zhang L, He T, et al. Cardiovascular implications of fatal outcomes of patients with coronavirus disease 2019 (COVID-19). JAMA Cardiol. 2020. https://doi.org/10.1001/ jamacardio.2020.1017.

13. Klok FA, Kruip M, Van der Meer NJM, Arbous MS, Gommers D, Kant KM, et al. Confirmation of the high cumulative incidence of thrombotic complications in critically ill ICU patients with COVID19: an updated analysis. Thromb Res. 2020;191:148-50. https:// doi.org/10.1016/j.thromres.2020.04.041.

14. Hill MA, Mantzoros C, Sowers JR. Commentary: COVID-19 in patients with diabetes. Metabolism. 2020;107:154217. https://doi. org/10.1016/j.metabol.2020.154217. 
15. Hussain A, Bhowmik B, do Vale Moreira NC. COVID-19 and diabetes: knowledge in progress. Diabetes Res Clin Pract. 2020;108142.

16. Gupta R, Ghosh A, Singh AK, Misra A. Clinical considerations for patients with diabetes in times of COVID-19 epidemic. Diabetes Metab Syndr. 2020;14(3):211-2.

17. Huang I, Lim MA, Pranata R. Diabetes mellitus is associated with increased mortality and severity of disease in COVID-19 pneumonia-a systematic review, meta-analysis, and meta-regression. Diabetes Metab Syndr Clin Res Rev. 2020;14(4):395-403.

18. Angelidi AM, Belanger MJ, Mantzoros CS. COVID-19 and diabetes mellitus: what we know, how our patients should be treated now, and what should happen next. Metab Clin Exp. 2020;107: 154245. https://doi.org/10.1016/j.metabol.2020.154245.

19. Cure E, Cure MC. Angiotensin-converting enzyme inhibitors and angiotensin receptor blockers may be harmful in patients with diabetes during COVID-19 pandemic. Diabetes Metab Syndr Clin Res Rev. 2020;14(4):349-50.

20. Fang L, Karakiulakis G, Roth M. Are patients with hypertension and diabetes mellitus at increased risk for COVID-19 infection? Lancet Respir Med. 2020;8:e21. https://doi.org/10.1016/S22132600(20)30116-8.

21. Baretić M. Case report of chloroquine therapy and hypoglycaemia in type 1 diabetes: what should we have in mind during the COVID19 pandemic? Diabetes Metab Syndr Clin Res Rev. 2020;14(4): 355-6.

Publisher's Note Springer Nature remains neutral with regard to jurisdictional claims in published maps and institutional affiliations. 